\title{
Informal Insurance, Enforcement Constraints, and Group Formation
}

\author{
Garance Genicot \\ Georgetown University \\ Debraj Ray \\ New York University and Instituto de Análisis Económico (CSIC)
}

We thank Fabien Moizeau for useful comments on an earlier draft. Address all correspondence to gg58@georgetown.edu and debraj.ray@nyu.edu. 


\section{Contents}

1. Introduction 2

2. Group Formation Under Equal Sharing 5

3. Stationary Transfers 8

4. General Results: Asymmetric Treatment and History Dependence 15

5. Some Final Remarks 18

$\begin{array}{ll}\text { References } & 20\end{array}$

$\begin{array}{ll}\text { Appendix } & 23\end{array}$ 


\section{Introduction}

This paper, largely based on Genicot and Ray (2003), discusses group formation in the context of informal insurance arrangements with enforcement constraints.

1.1. Risk-Sharing Agreements. Risk is a pervasive fact of life for most people, especially so in developing countries. A high and often extreme dependence on volatile labor markets or agricultural production, widespread poverty, and the lack of access to formal insurance and credit serve to create a particularly acute problem of consumption smoothing. It is not surprising, then, that formal insurance arrangements are supplanted by widespread informal arrangements. Such arrangements are not based on contracts that are upheld by a court of law but on the implicit promise of future benefits from continued participation and its attendant mirror image: the threat of isolation from the community as a whole in the event of noncompliance.

It hardly needs mentioning that there is considerable evidence of mutual insurance in village communities (Morduch (1991), Deaton (1992), Townsend (1994), Udry (1994), Jalan and Ravallion (1999), Ligon, Thomas and Worrall (2002), Grimard (1997), Gertler and Gruber (2002), and Foster and Rosenzweig (2002)). What is more interesting is that the same studies reveal a large departure from the ideal of perfect insurance. It is only natural to invoke various incentive constraints to explain the shortfall. Asymmetry of information, moral hazard and the lack of enforceability are all potential impediments to widespread risk-sharing.

Of these three factors, it appears that the most important constraint arises from the lack of enforceability of risk-sharing agreements. Udry (1994), for instance, finds this constraint to be the most important in describing the structure of reciprocal agreements in rural northern Nigeria. In the absence of explicit, legally binding contracts, these agreements must be designed to elicit voluntary participation. This constraint often seriously limits the extent of insurance informal risk-sharing agreements can provide.

Posner $(1980,1981)$ was the first to posit that voluntary risk-sharing can emerge between self-interested individuals if future reciprocity is expected. Following his insight, there has been a growing body of literature, both theoretical and empirical, on self-enforcing risk-sharing agreements. Some important theoretical contributions are Kimball (1988), Coate and Ravallion (1993), 
Kocherlakota (1996), Kletzer and Wright (2000), and Ligon, Thomas and Worrall (2002). All these studies define self-enforcing agreements as those that are proof from noncompliance by individual members of the group. ${ }^{1}$ According to the theory, the individual defector is isolated from the community, so that he must self-insure. With this insight in place, the common practice in the literature has been to define self-enforcing risk-sharing agreements as subgame perfect equilibria of a repeated game (in which self-insurance is always an option), and to characterize the Pareto frontier of such equilibria.

This kind of analysis has two important consequences. First, large groups always do better than smaller groups. Hence, efficient agreements have to be at the level of the "community".2 This is why most empirical tests of insurance take the unit of analysis as exogenous and study the extent of insurance at the level of the village or even larger groups. Second, a higher need for insurance, stemming for instance from a higher degree of risk aversion, relaxes the enforcement constraint and must therefore increase the extent of risk-sharing within a community.

1.2. Groups in Risk-Sharing. Our starting point is the following natural observation: If a large group - say, the village community or a particular caste or kin group within the community - can foresee the benefits of risk-sharing and reach an agreement, why can't smaller groups do the same? Indeed, one may go a step further and entertain the possibility that subgroups may agree to jointly defect and subsequently share risk among themselves. It follows that, to be truly self-enforcing, an informal risk-sharing agreement should be robust to joint deviations by subgroups.

At the same time, such group deviations must be themselves credible. To be of any value, or to pose a credible threat to the group at large, a deviating coalition should also employ selfenforcing arrangements. These embedded constraints characterize the concepts of self-enforcing risk-sharing agreements and stable coalitions that we define in Genicot and Ray (2003). We study group formation in informal insurance within communities, recognizing that not just the

\footnotetext{
${ }^{1}$ In the words of Telser (1980, p.27), "In a self-enforcing agreement each party decides unilaterally whether he is better off continuing or stopping his relation with the other parties".

${ }^{2}$ Of course, considerations of asymmetric information or some other cost of group formation may close off group size before the community limit is reached. See below for further discussion of this point.
} 
extent of insurance within a given group is endogenous, but that this affects and is affected by the process of group formation itself.

This has two important implications that sharply contrast with the individual-deviation model. First, subgroups of individuals may destabilize insurance arrangements among the larger group, thereby limiting group size. Second, an increase in the need for insurance - stemming either from a change in the environment or in some behavioral parameter, such as the degree of risk aversion - can actually decrease the extent of risk-sharing among the population, by reducing the maximal stable group size.

Indeed, the few papers that address the issue of risk-sharing among subgroups actually find convincing evidence for the existence of subgroups. Lomnitz [1977] finds that reciprocity networks in Cerrada del Cóndor, a shanty-town of about 200 dwellings in the southern sector of Mexico City, are composed of an average of 3.65 nuclear families. Fafchamps and Lund (2003) address a similar question in the context of the rural Philippines. While gifts and loans circulate among networks of friends and relatives, risk is far from efficiently shared at the village level. Likewise, Murgai, Winters, Sadoulet and DeJanvry (2002) investigate water transfers among households along a watercourse in Pakistan's Punjab and find that reciprocal exchanges are localized in units smaller than the entire watercourse community.

To be sure, there are other potential explanations for observed limits on group size. Geographical proximity (or lack thereof), the limited observability of actions or types, a varying ability to punish slackers, or positive covariance in the income distribution: all these factors can explain differences in the extent of insurance, with some clusters of individuals making more transfers to each other than to others. However, except in extreme cases, all agents would be expected to transact with each other directly or indirectly, at least to some extent. Murgai et al. suggest that the explanation for the formation of these subgroups must lie in the existence of setup costs that with the number of participants in the risk-sharing agreement: "If establishing and maintaining partnerships is indeed costless, there is no reason for a mutual insurance group not to be community-wide or world-wide. Real world limits to group size must therefore be the result of costs relating to the formation and maintenance of partnerships" (Murgai et al. (2002), 
p.251). However, this paper suggests that there may be more fundamental reasons for group splintering.

1.3. Outline of Paper. In what follows, we illustrate the group formation question by means of the simplest possible model (Section 2). In this setup, a group that forms must insure each other to the maximal extent possible (we call this the equal sharing norm). Adherence to such a norm at the group level does not, of course, do away with the enforcement constraint. Splinter subgroups (conceivably individuals but often nondegenerate groups) may well break off from the larger group. Subsequently they, too, must follow the equal-sharing group, and their stability will be tested in exactly the same way.

In Section 3, we extend the model to allow for the recognition (by a group) that it may be constantly under threat from potential deviants. Such recognition will generally entail a departure from equal sharing, with more limited transfers. To be sure, in the interests of consistency, we must permit a similar self-exploration on the part of deviant subgroups. Thus, as we expand the possibilities for the group as a whole, we also expand the range of threats to its stability. Finally, in Section 4, we comment on a further widening of insurance schemes to include history-dependent quasi-credit.

The emphasis throughout this paper is on specific examples rather than on full generality. Readers invited in the details of a more general analysis are invited to consult Genicot and Ray (2003).

\section{Group Formation Under Equal Sharing}

A community of $n$ identical agents engages in the production and consumption of a perishable good at each date. Each agent produces a random income that is high $h$ with probability $p$ and low $\ell$ with probability $1-p$. Income realizations are independent and identical, over people as well as dates. Each agent has the same utility function, increasing, smooth and strictly concave in consumption. They discount future at a rate $\delta \in(0,1)$.

Consider any grouping of individuals in this community, and suppose that its members are currently pledged to mutually insure one another against consumption fluctuations. We assume that such insurance is to the maximum extent possible: group output is shared equally among all 
the members. We refer to this practice as equal sharing. [It is obvious that this is the first-best symmetric scheme.]

Let $\widetilde{v}(n)$ denote the expected utility from the equal sharing scheme. When $k$ individuals draw $h$, all group members consume $\frac{k}{n} h+\frac{n-k}{n} \ell$. This implies a per-period expected utility of

$$
\widetilde{v}(n) \equiv \sum_{k=0}^{n} p(k, n) u\left(\frac{k}{n} h+\frac{n-k}{n} \ell\right)
$$

where $p(k, n)$ is just the probability of $k$ highs out of $n$ draws. $^{3}$

Equal-sharing stability may be defined recursively as follows. By definition, singletons or individuals are equal-sharing stable and the worth of a singleton group is just $\tilde{v}(1)$. Recursively, having assessed equal-sharing stability for all $m=1, \ldots, n-1$, a coalition of size $n$ is said to be equal-sharing stable if, for all $k=1, \ldots, n-1$,

$$
(1-\delta)\left[u(h)-u\left(\frac{k}{n} h+\frac{n-k}{n} \ell\right)\right] \leq \delta(\tilde{v}(n)-\tilde{v}(s))
$$

for every equal-sharing stable $s \leq k$. This constraint requires that the short term deviation gain from not making the transfer, on the left-hand-side, be smaller than the long term gain from remaining in the risk sharing agreement rather than deviating in a group of size $s$, on the right-hand-side. If $n$ is equal-sharing stable then its worth is simply $\tilde{v}(n)$. Note that for a given equal-sharing stable size $s$ it actually suffices to check the constraint for $k=s$ since the left-hand side is decreasing in $k$.

Proposition 1. Independently of the overall community size, there is a finite upper bound on the equal-sharing stable sizes.

It is easy to see why (see formal proof in appendix). If the assertion were false, there would be an infinity of stable sizes. But we do know that the marginal "diversification gain" from an increase in size ultimately tends to zero. Therefore, for a very small $\epsilon$, we may pick a stable size $n$ such that a coalition of size $n$ is able to reap most of the benefits of sharing risk: a larger stable group improves the per-capita utility of its members by no more than $\epsilon$. Because the set

$\overline{3^{3} \text { That is, } p(k, n)}=\frac{n !}{k !(n-k) !} p^{k}(1-p)^{n-k}$. 
of stable sizes is infinite, we can choose a stable coalition sufficiently larger than $n$ such that the short term gain of deviating from this coalition when $n$ agents have a good shock is strictly larger than the relative long term gain from being in this larger coalition rather than in a group of $n$.

Moreover, it is possible to show that for a large range of preferences the set of equal-sharing stable sizes is a "connected" set of integers. To identify this range, consider the following condition:

[QC] For every $k,(1-\delta) u\left(\frac{k}{n} h+\frac{n-k}{n} \ell\right)+\delta \widetilde{v}(n)$ is quasi-concave in $n$ for all $n \geq k$.

Condition $[\mathrm{QC}]$ is satisfied for several utility functions. It is true, for instance, for all utility functions exhibiting a relative risk aversion of at least 2 , as well as for quadratic or cubic preferences. Now we may state the following proposition (see appendix for proof):

PROPOSITION 2. For all utility functions satisfying $[\mathrm{QC}]$, if a group of size $n$ is not equalsharing stable then a group of size $n^{\prime}>n$ is not equal-sharing stable either.

For instance, with a utility function given by $u(x)=-\frac{1}{2}(B-x)^{2}$ for some $B>h$ it is possible to show that $n$ is stable if and only if for every $1 \leq k \leq n-1$,

$$
\frac{C}{k}+\frac{k}{n} \geq \frac{2}{\theta}+1
$$

where $C=\frac{\delta}{1-\delta} p(1-p)$ and $\theta=\frac{h-\ell}{B-h}$ (this latter variable will later reappear as our proxy for the need for insurance).

From the inequalities (0.3), it is easy to see that for the same $k$ and $n$ a mean preserving spread in the income distribution (higher $\theta$ ) and a higher patience $\delta$ relax the constraints. Hence, these increase (or at least leave unchanged) the set of equal-sharing stable sizes. Similarly, higher values of $p(1-p)$ ( $p$ closer to $1 / 2)$ correspond to a higher variance and therefore, if anything, increase the set of stable sizes.

Condition (0.3) may also be used to obtain a tighter description of the maximal equal-sharing stable group. We illustrate this by neglecting integer constraints (which are easily accounted for). Observe that the left-hand side of (0.3) is minimized (in $k$ ) when $k=\sqrt{n C}$, this condition being 
applicable when $n>C$. Solving for the minimum value, we see that the maximal group size $M$ is bounded above by the inequality

$$
M \leq \max \left\{C, \frac{4 C}{\left(\frac{2}{\theta}+1\right)^{2}}\right\} .
$$

Notice that $M$ is bounded uniformly in $\theta$. With our later interpretation of $\theta$ as a measure of the need for insurance, this means that maximal stable size cannot rise indefinitely in need.

\section{Stationary Transfers}

To be sure, even when an equal-sharing agreement is not possible, individuals may be able to design a risk-sharing agreement by limiting transfers in states for which the enforcement constraint is binding. Kimball (1988) and Coate and Ravallion (1993) study the best stationary risk-sharing agreements. In this section, we emulate the approach of these authors to find the best constrained risk-sharing agreement. At the same time, we also bear in mind that groups as well as individuals may deviate. In short, we develop the theory of group enforcement constraints under the assumption that each coalition or group, once formed, attempts to implement some symmetric and stationary risk-sharing arrangement.

As in the previous section, group stability is defined recursively. Once again, individuals (or singleton coalitions) are stable. The lifetime utility of an individual in isolation (normalized by the discount factor to a per-period equivalent) is simply

$$
v^{*}(1) \equiv p u(h)+(1-p) u(\ell) .
$$

This is the stable worth of a "singleton group".

Recursively, having defined stability (and stable worths) for all $m=1, \ldots, n-1$, consider some coalition of size $n$. We first define a (symmetric and stationary) transfer scheme. This may be written as a vector $\mathbf{t} \equiv\left(t_{1}, \ldots, t_{n-1}\right)$, where $t_{k}$ is to be interpreted as the (nonnegative) transfer or payment by a person in the event that his income is $h$ and $k$ individuals draw $h$. We only consider nontrivial schemes in which $t_{k}>0$ for some $k$.

With a transfer scheme in mind we can easily back out what a person receives if his income draw is $\ell$ and $k$ individuals produce $h$. The total transfer is then $k t_{k}$, to be divided equally among 
the $n-k$ individuals who produce $l$. Thus a transfer scheme $\mathbf{t}$ implies the following: if there are $k$ high draws, then a person consumes $h-t_{k}$ if he produces $h$, and $\ell+\frac{k t_{k}}{n-k}$ if he produces $\ell$. It follows that the expected utility from a transfer scheme $\mathbf{t}$ is given by

$$
v(\mathbf{t}, n) \equiv p^{n} u(h)+(1-p)^{n} u(\ell)+\sum_{k=1}^{n-1} p(k, n)\left[\frac{k}{n} u\left(h-t_{k}\right)+\frac{n-k}{n} u\left(\ell+\frac{k t_{k}}{n-k}\right)\right],
$$

where $p(k, n)$ - as before - is the probability of $k$ highs out of $n$ draws. Define a (nontrivial) transfer scheme $\mathbf{t}$ to be stable if for all $k=1, \ldots, n-1$,

$$
(1-\delta) u\left(h-t_{k}\right)+\delta v(\mathbf{t}, n) \geq(1-\delta) u(h)+\delta v^{*}(s)
$$

for every stable $s \leq k$.

The interpretation of stability is quite simple. We require that for all possible income realizations, the stipulated transfers actually be carried out. If inequality (0.6) fails for some $k$ and $s \leq k$, this means that there is a stable coalition of size $s$ that would rather refuse to pay what they are required to pay (when $k$ individuals draw high) and share risk with each other such that this transfer would actually not be made.

It is useful to compare our definition of self-enforcing insurance with the one used in the literature in which only the individual enforcement constraint must be respected. We shall call this individual stability, or $i$-stability for short. Fix a population of $n$ individuals, and let $\widehat{v}(n)$ denote the maximum value of (0.5) when (0.6) is only invoked for $s=1$. In other words, $\widehat{v}(n)$ is the solution to the following problem

$$
\max _{\mathbf{t}} v(\mathbf{t}, n)
$$

subject to

$$
(1-\delta) u\left(h-t_{k}\right)+\delta v(\mathbf{t}, n) \geq(1-\delta) u(h)+\delta v^{*}(1)
$$

for all $k=1, \ldots, n-1$.

If there is some nontrivial transfer scheme that solves this problem, say that a group of size $n$ is i-stable. 
In what follows, we will show that the concepts of stability and i-stability have very different implications for the extent of insurance that we expect a community to achieve. For this purpose, it will be useful to measure the need for insurance as the ratio $\frac{u^{\prime}(\ell)-u^{\prime}(h)}{u^{\prime}(h)}$, which we henceforth denote by $\theta$. [The reader can check that this definition reduces precisely to the corresponding $\theta$ in the example of the previous section.] Keeping everything else constant, a mean preserving spread between $h$ and $\ell$ increases $\theta$. Moreover, for the same income distribution, a utility function that exhibits a higher risk aversion throughout its domain will translate into a higher need for insurance. Hence, our measure incorporates both environmental uncertainty and attitudes towards risk, albeit in summary form.

We now proceed in further characterizing the sets of i-stable and stable sizes.

First, notice that if a group is not i-stable it cannot be stable since stability only adds constraints to the problem. Hence, the set of stable size is included in the set of i-stable sizes.

Next, note from inequality (0.8) that for the same transfer to be made today, and therefore the same short-term gain from deviating, being in a larger group means larger long-term benefits. This implies that if a group size is i-stable, any larger size must be stable too. In particular, in Genicot and Ray (2003) we show that a group size $n$ is i-stable if and only if

$$
\theta>\frac{1-\delta}{\delta p\left(1-p^{n-1}\right)}
$$

Clearly, the right-hand-side of (0.9) is decreasing in $n$ and bounded below by $\underline{\theta} \equiv \frac{1-\delta}{\delta p}$. It follows that, if the need for insurance $\theta$ is no larger than $\underline{\theta}$, there is no i-stable or stable risksharing agreement and only autarky is possible. In contrast, if $\theta>\underline{\theta}$ not only is the set of i-stable sizes non-empty but it is infinite. The smallest i-stable group size is the smallest value of $n$ such that (0.9) holds and any larger size is i-stable too.

Now suppose that $\theta>\underline{\theta}$. What can we say about the set of stable sizes? Consider the smallest i-stable size. Since no smaller size is stable, no deviations other than individual deviations are credible. Hence, the same risk-sharing agreement is stable and this group size is also the smallest stable group size. With larger group sizes, however, this argument breaks down. Once groups of intermediate size are stable, they begin to pose credible threats to groups of larger size, and i-stability no longer implies stability. 
This raises the question of whether there can be an infinite number of stable groups. Proposition 1 suggests the answer is no, but because we are no longer restricted to the equal-sharing norm the argument here is more subtle. In Genicot and Ray (2003) we show that, indeed, only a finite number of sizes can be stable and therefore that the set of stable sizes has a finite upper bound. To see why this must be true assume as earlier than the assertion is false and therefore that there is an infinity of stable sizes. As before, we can then pick a stable size $n$ such that a coalition of size $n$ is able to reap most of the benefits of sharing risk: a larger stable group improves the per-capita utility of its members by only a small amount. The difference with equalsharing stability is that now one can limit the transfer to satisfy the enforcement constraints. This means that in any larger stable coalition, the transfers made whenever at least $n$ people have a good shock have to be close to 0 . But, because the set of stable sizes is infinite, we can choose this stable coalition sufficiently large such that the probability of at least $n$ people having a good shock is close to 1 . This implies that the worth of such a coalition can be brought arbitrarily close to autarkic utility, but this contradicts the presumed stability of that coalition.

The above findings are summarized in the following proposition (proof omitted) and illustrated in Figure 0.1.

Proposition 3. [Genicot and Ray (2003)] For each level of need for insurance $\theta>\frac{1-\delta}{\delta p}$, there are thresholds $\bar{n}(\theta)$ and $\underline{n}(\theta)$ such that

$$
2 \leq \underline{n}(\theta) \leq n \leq \bar{n}(\theta)<\infty,
$$

for every stable group size $n$. Moreover,

$$
\underline{n}(\theta) \uparrow \infty \text { as } \theta \downarrow \frac{1-\delta}{\delta p} .
$$

In addition, from Proposition 3 follows a general "nonmonotonicity" result: a higher need for insurance can actually translate into lower group sizes. This can been seen directly from Figure 0.1. Pick a community size $\tilde{n}$ that lies above the "stable correspondence" at some point. Then for any $n \geq \tilde{n}$, we can find two degrees of uncertainty $\theta_{1}$ and $\theta_{2}$, with $\theta_{2}>\theta_{1}$, such that $n$ is 


\section{Figure 0.1. An Illustration of Proposition 3.}

stable under $\theta_{1}$ but not under $\theta_{2}$. It is easy enough to pick $\theta_{1}$ to be such that $n$ is the smallest stable size that can be supported under $\theta_{1}$, and pick $\theta_{2}$ such that the largest stable size under $\theta_{2}$ is strictly less than falls below $n$. Hence, the non-monotonicity.

The following example from Genicot and Ray (2003) illustrates well the main features of our concept of stability.

EXAMPLE. Consider a community of ten individuals with a constant relative risk aversion utility function:

$$
u(c)=\frac{1}{1-\rho} c^{1-\rho},
$$

where $\rho$ is the Arrow-Pratt coefficient of relative risk aversion. Assume the following values for the specific parameters: $\delta=0.83, \rho=1.6, p=0.4, \ell=2$, and $h=3$.

We evaluate - for each group size ranging from 1 to 10 - the return to informal insurance. One natural way to do this is to look at the gain over and above autarky, compared to the corresponding per-capita gain that the first-best provides in the community of all ten. If $\tilde{v}$ denotes this latter value and $\hat{v}(n)$ is the i-stable value for a group of size $n$, then the $i$-stable gain 


\begin{tabular}{cccc}
\hline$n$ & Stable? & i-Stable Gain (\%) & Stable Gain (\%) \\
\hline 1 & $\sqrt{ }$ & 0 & 0 \\
2 & $\sqrt{ }$ & 10 & 10 \\
3 & $\sqrt{ }$ & 50 & 38 \\
4 & $\times$ & 61 & $\emptyset$ \\
5 & $\times$ & 69 & $\emptyset$ \\
6 & $\times$ & 75 & $\emptyset$ \\
7 & $\times$ & 78 & $\emptyset$ \\
8 & $\times$ & 81 & $\emptyset$ \\
9 & $\times$ & 84 & $\emptyset$ \\
10 & $\times$ & 85 & \\
\hline
\end{tabular}

Table 0.1. Stable Gains are Limited.

may be reported as

$$
\frac{\hat{v}(n)-v(1)}{\tilde{v}-v(1)} \times 100
$$

in percentage terms. Similarly, if $v^{*}(n)$ is the stable value for a group of size $n$, then the stable gain is described as

$$
\frac{v^{*}(n)-v(1)}{\tilde{v}-v(1)} \times 100,
$$

again in percentage terms. The results for this example are reported in Table 0.1.

It turns out that within this population of 10 and for the parameter values described, only individuals and groups of size 2 and 3 are stable. The question arise then: which groups do we expect to see and if there are groups of different sizes which payoffs do we look at? Since we are looking at constrained efficient schemes among identical agents, a good contender is the partition of the population into stable groups that maximizes the expected utility of an agent, under the assumption that his probability to be in any given group is proportional to the size of the group. In this example, this rule predicts that the population would break into three stable groups of three individuals each and one singleton group (which means a $90 \%$ chance of getting $v^{*}(3)$ and a $10 \%$ chance of obtaining $v(1)$ ). That is, an individual's stable payoff gain is $38 \%$ (see Table 0.1 ) with probability $9 / 10$ and 0 otherwise. This implies a stable gain of only $34 \%$ which is less than half the return $(85 \%)$ were we not to account for coalition formation.

One might object that Example 2 is only described for a very special set of parameter values. So, to build Table 0.2 we took similar parameters but consider different values for the probability 


\begin{tabular}{cccc}
\hline$p \backslash \theta$ & .75 & 1 & 2 \\
\hline .2 & 1 & 1 & 1,2 \\
.4 & 1,3 & 1,2 & 1,2 \\
.6 & 1,3 & $1,2,5$ & 1,2 \\
.8 & $1,3,5$ & 1,3 & $1,2,7$ \\
\hline
\end{tabular}

Table 0.2. Stable Sizes

of a high income $p$ and for the need for insurance $\theta$. Table 0.2 reports the stable group sizes for the different values of $p$ and $\theta$.

To summarize the discussion so far, we see that

(1) The stability of smaller sizes may impede the stability of larger sizes.

(2) Much has been written on "social capital" in the past few years. In the insurance context one could measure the return to such capital very much as we have done here. However, recognizing the possibility of coalition deviations dramatically reduces the estimated return on social capital.

(3) Computations for several parameter values reveal both robustness and sensitivity, in the following senses. Changing the parameters even slightly causes some stable sizes to become unstable and vice versa as Table 0.2 illustrates. Yet the results are surprisingly robust in the sense that potential coalitional deviations inevitably cause a large fraction of the potential benefits from insurance not to be reaped.

(4) Moreover, at $p=0.4$ if the need for insurance $\theta$ increases from 0.75 to 1 the largest stable size decreases from 3 to 2 . This suggests that even an increase in risk may destroy previously successful insurance arrangements as previously non-viable subgroups now become viable, destroying the viability of the larger community.

One can extend this analysis to heterogeneous agents. Heterogeneity is a natural feature of life and is likely to play an important role in group formation. Kinship, family, clan and religious affiliation are important in this respect, because they help in punishing and imposing strict norms on members (Platteau (1991) and Fafchamps (1992)). At the same time, characteristics that strengthen risk sharing between some individuals will tend to worsen the performance of 
risk-sharing agreements within a larger group or even destroy altogether the stability of these larger groups.

We illustrate this with a simple example. Consider two households each composed of two members. We shall assume that households have means of punishing their own members who would defect from a risk-sharing agreement but not individuals outside their households. Assume logarithmic utility and the following values for the parameters: $\ell=1, h=1.488, p=0.5$ and $\delta=0.9$. The appendix shows that, in this case, the ability of individuals to perfectly share risk within their household prevents them from sharing risk across households. Indeed, no stationary insurance agreement can be designed involving positive transfer between the two households. As a result, individuals enjoy a utility of $\widetilde{v}(2)$ per period. However, in the absence of any enforcement power within households, these individuals would be able to all share risk with each other and enjoy $v^{*}(4)$. This, although still imperfect, would represent a $16 \%$ increase in the gain from insurance! ${ }^{4}$

\section{General Results: Asymmetric Treatment and History Dependence}

The last section went a long way towards endogenizing both group formation and risk-sharing agreements. However, the latter were restricted to be symmetric and stationary. In this section, we explain why risk-sharing agreements will generally make use of history dependence and of asymmetric strategies in subgames, and discuss the robustness of our results.

The reader familiar with the recent literature on insurance knows that when the lack of enforcement limits risk-sharing the optimal i-stable risk-sharing agreement is non-stationary. The reason is simple. With stationary transfers, all agents face the same continuation utility regardless of their current shock. However, when perfect risk-sharing is not possible, an agent suffering from a low shock today has a higher marginal utility than an agent with a high income. It follows that there exists a mutually beneficial modification in the agreement in which the lowincome agent trades off some of his continuation utility in exchange for a slightly higher transfer today. The low-income agent promises to make some transfer (within the self-enforcing range) to the high-income agent in the event that both of them draw a high or a low shock next period.

${ }^{4}$ The gain is evaluated as $\frac{v^{*}(4)-\widetilde{v}(2)}{\widetilde{v}(4)-v(1)} \times 100$. 
Thus the efficient scheme tends to equalize the inter-temporal rates of substitution between the agents. As Kocherlakota (1996) showed, all the history dependence of the optimal i-stable risksharing agreement is captured in the ratios of marginal utility of the agents. Given last period's ratio of marginal utilities, the continuation expected utilities of the agents follows a stationary distribution. Because the ratio of marginal utilities is one possible measure of the agents' relative needs, the structure of history-dependent schemes fits the empirical evidence well.

Numerous studies in the economic and anthropological literature provide evidence that informal risk-sharing agreements and informal credit arrangements are not clearly separated (see, for instance, Evans-Pritchard (1940), Platteau and Abraham (1987) and Udry (1994)). These studies report a large reliance on what is observationally equivalent to informal loans with an implicit repayment scheme contingent on the lender's needs and the borrower's ability to repay. Ligon, Thomas and Worrall (2002), Foster and Rosenzweig (2001) and Fafchamps and Lund (2001) all find that the history-dependent scheme fits the data better than the benchmark of perfect risk-pooling and the stationary limited commitment model.

Now, a particular source of history-dependence and asymmetry stems specifically from our consideration of group formation. When group deviations are important, symmetry necessitates that we compensate all potential deviants in a subgroup in order to prevent a deviation. If that symmetry is broken, then not all deviants need to be so treated. A subset, containing the minimal number that must be compensated in order to avoid the deviation, will suffice. For instance, to prevent two individuals from deviating, the scheme may require a sufficiently low transfer from one of them so that he would not participate in a joint deviation, while demanding from the other a higher transfer (but not too high, so that individual deviations are still not worthwhile). This will be the case even if both agents experienced the same history of shocks. Clearly, randomization will play an important role in this case to decide who gets to make the lower transfer. [Randomization would retain symmetry ex ante.]

With these asymmetries, it is no longer necessary to compensate every member of every potential subgroup; it is only necessary to compensate some member of every potential subgroup. In contrast, in a symmetric and stationary equilibrium, every member of a potentially deviant 
subgroup must be simultaneously compensated for staying with the ambient group. Such compensations become impossible because the marginal gains to group size vanish, whereas deviation gains are bounded away from zero, precipitating the boundedness result in the stationary case. In the general case, it is possible to switch carefully to asymmetric strategies following appropriate histories of good and bad draws.

To allow for history-dependence, asymmetries, as well as randomization, insurance schemes need to be redefined. For a group of size $n$, let $\mathbf{y}$ be a vector of realized incomes; that is, $y_{i}$ is either $h$ or $\ell$ for each $i=1, \ldots, n$. Let $\mathbf{c}$ be a nonnegative vector of consumptions. Say that $\mathbf{c}$ is feasible (under $\mathbf{y}$ ) if $\sum_{i} c_{i}=\sum_{i} y_{i}$. For any date $s$, an $s$-history - call it $H_{s}$ - is a list of all past income realizations and (feasible) consumption vectors. [At $s=0$, simply use any singleton to denote the 0-history.]

Define $\mathcal{M}(\mathbf{y})$ to be the set of all probability measures over consumption vectors $\mathbf{c}$ such that $\mathbf{c}$ is feasible for $\mathbf{y}$. An insurance arrangement is a list of functions $\sigma=\left\{\sigma_{s}\right\}_{s=0}^{\infty}$ such that for all $s \geq 0, \sigma_{s}$ maps the product of $s$-histories and current income realizations $\mathbf{y}$ to lotteries in $\mathcal{M}(\mathbf{y})$. We'll say that an insurance arrangement is nontrivial if it places positive probability on schemes that involve nonzero transfers for some states.

Note that an insurance arrangement generates a vector of expected payoffs following every $s$ history $H_{s}$ : call this vector $\mathbf{v}\left(H_{s}, \sigma, n\right)$. [These are discounted normalized expected payoffs for each individual in the group, before the realization of current incomes and, of course, the consumption lottery.]

To define stability, we proceed recursively just as in the stationary case. Individuals (or singleton coalitions) are automatically branded stable. Indeed, there is only one stable payoff for an "individual coalition", which is just the no-insurance payoff at every date. That is, if we define

$$
v^{*}(1) \equiv p u(h)+(1-p) u(\ell)
$$

then the set of stable payoffs is just $V^{*}(1) \equiv\left\{v^{*}(1)\right\}$. 
Now suppose that we have defined stable payoff sets $V^{*}(m)$ for all $m=1, \ldots, n-1$ (some of these may be empty). Pick a group of size $n$ and a nontrivial insurance arrangement $\sigma$ for this group. Say that $\sigma$ is stable if the following two conditions are satisfied:

[PARTicipation.] For no $s$-history $H_{s}$ is there a subgroup of individuals (of size $m<n$ ) and a stable payoff vector $\mathbf{v} \in V^{*}(m)$ such that $v_{i}\left(H_{s}, \sigma, n\right)<v_{i}$ for all $i=1, \ldots, m$.

[EnForcement.] The following is a zero-probability event under $\sigma$ : there is an $s$-history $H_{s}$, an income realization $\mathbf{y}$, and a prescribed consumption allocation $\mathbf{c}$ such that for some subgroup of individuals (of size $m<n$ ) and some stable payoff vector $\mathbf{v} \in V^{*}(m)$,

$$
(1-\delta) u\left(y_{i}\right)+\delta v_{i}>(1-\delta) u\left(c_{i}\right)+\delta v_{i}\left(H_{s+1}, \sigma, n\right)
$$

where $H_{s+1}$ is the $(s+1)$-history obtained by concatenating $H_{s}$ with $\mathbf{y}$ and $\mathbf{c}$.

If $\sigma$ is stable, then say that $\mathbf{v}\left(h_{0}, \sigma, n\right)$ is a stable payoff vector for $n$. If no such vector exists, we say that $n$ is unstable and set $V^{*}(n)$ to the empty set.

In Genicot and Ray (2003) we show that our main result extends to this fully general case. For every value of $\theta$ such that some stable group exists, the maximal stable group size is finite.

\section{Some Final Remarks}

We end with two remarks, one methodological and one specific to the study of insurance arrangements.

The reader familiar with the recent literature on endogenous coalition formation (see, e.g., Bloch (1996), and Ray and Vohra $(1997,1999,2001))$ will see the close parallel to our approach. However, there is one important difference. In the literature on endogenous coalition formation, coalitions respond to a proposed ex ante arrangement. That is, coalitional constraints are evaluated at the level of the "participation constraints". In contrast, in this paper, coalitions respond after learning the realization of income shocks at every date, and after learning what their actions are to be. These ex-post considerations are closer to "incentive constraints." In this sense, our approach also bears a connection to the coalition-proof Nash equilibrium (Bernheim, Peleg and Whinston (1987)). 
Moreover, the existence of an upper bound on stable groups is in stark contrast with the existence of infinitely many stable sizes in the coalition formation literature (see, e.g., Bloch (1996) and Ray and Vohra (1997) for results on stable cartels in oligopoly, and Ray and Vohra (2001) for results on the efficient provision of public goods). It is is peculiar to the insurance problem. 


\section{References}

(1) Bernheim, D., B. Peleg and M. Whinston (1987), "Coalition-proof Nash equilibria. I. concepts," Journal of Economic Theory 42, 1-12.

(2) Bloch, F. (1996), "Sequential Formation of Coalitions with Fixed Payoff Division," Games and Economic Behavior 14, 90-123.

(3) Coate, S. and M. Ravallion (1993), "Reciprocity without Commitment: Characterization and Performance of Informal Insurance Arrangements," Journal of Development Economics 40, 1-24.

(4) Deaton, A. (1992), Understanding Consumption. Oxford: Clarendon Press.

(5) Evans-Pritchard, E. (1940), The Nuer: A Description of the Modes of Livelihood and Political Institutions of a Nilotic People, Oxford : Clarendon Press.

(6) Fafchamps, M. (1992) "Solidarity Networks in Preindustrial Societies: Rational Peasants with a Moral Economy," Economic Development and Cultural Change 41, 147-174.

(7) Fafchamps, M. (1996), "Risk Sharing, Quasi-Credit, and the Enforcement of Informal Contracts," mimeo., Department of Economics, Stanford University.

(8) Fafchamps, M. and S. Lund (2003), "Risk Sharing Networks in Rural Philippines." Journal of Development Economics 71, 233-632.

(9) Foster, A. and M. Rosenzweig (2001), "Imperfect Commitment, Altruism, and the Family: Evidence from Transfer Behavior in Low-Income Rural Areas," Review of Economics and Statistics 83, 389-407.

(10) Genicot, G. and Ray, D. (2003), "Endogenous group formation in risk-sharing arrangements," Review of Economic Studies 70, 87-113.

(11) Gertler, P. and J. Gruber (2002), "Insuring Consumption Against Illness," American Economic Review 92, 51-70.

(12) Grimard, F. (1997), "Household Consumption Smoothing through Ethnic Ties: Evidence from Côte D'Ivoire," Journal of Development Economics 53, 391-422.

(13) Jalan, J. and M. Ravallion (1999), "Are the Poor Less Well Insured? Evidence on Vulnerability to Income Risk in Rural China," Journal of Development Economics 58, $61-81$. 
(14) Kimball, M. (1988), "Farmer Cooperatives as Behavior Toward Risk," American Economic Review 78, 224-232.

(15) Kletzer, K. and B. Wright (2000), "Sovereign Debt as Intertemporal Barter," American Economic Review 90, 621-639.

(16) Kocherlakota, N. (1996), "Implications of Efficient Risk Sharing without Commitment," Review of Economic Studies 63, 595-609.

(17) Ligon, E., Thomas, J. and T. Worrall (2002), "Mutual Insurance and Limited Commitment: Theory and Evidence in Village Economies," Review of Economic Studies 69, $115-139$.

(18) Lomnitz, A.L. (1977), Networks and Marginality : Life in a Mexican Shantytown. New York : Academic Press.

(19) Morduch, J. (1991) "Consumption Smoothing Across Space: Tests for Village Level Response to Risk," Harvard University, mimeo.

(20) Murgai, R., Winters, P., Sadoulet, E. and A. de Janvry (2002), "Localized and Incomplete Mutual Insurance," Journal of Development Economics 67, 245-274.

(21) Platteau, J-Ph. (1991), "Traditional Systems of Social Security and Hunger Insurance: Past Achievements and Modern Challenges," in Social Security in Developing Countries, edited by E. Ahmad, J. Dreze, J. Hills, and A. Sen, Oxford: Clarendon Press.

(22) Platteau, J-Ph. and A. Abraham (1987), "An Inquiry into Quasi-credit Contracts: The Role of Reciprocal Credit and Interlinked Deals in Small-scale Fishing Communities," Journal of Development Studies 23, 461-490.

(23) Posner, R. (1980), "A Theory of Primitive Society, with Special Reference to Law," Journal of Law and Economics 23, 1-53.

(24) Posner, R. (1981), The economics of justice. Harvard University Press.

(25) Ray, D. and R. Vohra (1997), "Equilibrium Binding Agreements," Journal of Economic Theory $73,30-78$.

(26) Ray, D. and R. Vohra (1999), "A Theory of Endogenous Coalition Structures," Games and Economic Behavior 26, 286-336. 
(27) Ray, D. and R. Vohra (2001), "Coalitional Power and Public Goods," Journal of Political Economy 109, 1355-1384.

(28) Telser, L. (1980), "A theory of self-enforcing agreements," Journal of Business 53, 27-44.

(29) Thomas, J. and T. Worrall (2002), "Gift-giving, quasi-credit and reciprocity," Rationality and Society, 14(3), 307-351.

(30) Townsend, R. (1994), "Risk and Insurance in Village India," Econometrica 62, 539-591.

(31) Udry, C. (1994), "Risk and Insurance in a Rural Credit Market: An Empirical Investigation in Northern Nigeria," Review of Economic Studies 61, 495-526. 


\section{Appendix}

Proof of Proposition 1. Suppose that Proposition 1 is false. Then there exists an infinite set $\mathcal{N}$ such that for all $n \in \mathcal{N}, n$ is stable. Since $\widetilde{v}(n)$ is increasing, if $n$ and $n^{\prime}$ are both in $\mathcal{N}$ and $n<n^{\prime}$, then $\widetilde{v}(n) \leq \widetilde{v}\left(n^{\prime}\right)$. Moreover, $\{\widetilde{v}(n)\}_{n \in \mathcal{N}}$ is bounded. It follows that for any $\epsilon>0$, there exists $n(\epsilon) \in \mathcal{N}$ such that for all $n \in \mathcal{N}$ with $n>n(\epsilon)$,

$$
\widetilde{v}(n)-\widetilde{v}(n(\epsilon))<\epsilon
$$

Moreover, it is easy enough to choose $n(\epsilon)$ satisfying both $(0.14)$ and the requirement that

$$
\epsilon<\frac{1-\delta}{\delta}[u(h)-u(\ell)]-A
$$

for some $A>0$. Now consider some stable $n>n(\epsilon)$. It is obvious that as $n \rightarrow \infty$,

$$
u(h)-u\left(\frac{n(\epsilon)}{n} h+\frac{n-n(\epsilon)}{n} \ell\right) \rightarrow u(h)-u(\ell) .
$$

It follows that as $n \rightarrow \infty$,

$$
u(h)-u\left(\frac{n(\epsilon)}{n} h+\frac{n-n(\epsilon)}{n} \ell\right)>\widetilde{v}(n)-\widetilde{v}(n(\epsilon))
$$

which contradicts the stability of $n$.

Proof of Proposition 2. For any $k$ and any $n \geq k$, define

$$
I(k, n) \equiv-(1-\delta)\left[u(h)-u\left(\frac{k}{n} h+\frac{n-k}{n} \ell\right)\right]+\delta(\tilde{v}(n)-\tilde{v}(k))
$$

Invoking $[\mathrm{QC}]$, it is easy to see that $I(k, n)$ must be quasi-concave in $n$. Because $I(k, k)=0$, the quasi-concavity of $I(k, n)$ implies that if $I(k, n)<0$ for any $n>k$, then the same must be true of any $n^{\prime}>n$.

Details of the final example in Section 3. Within a household, perfect risk-sharing can be achieved. This implies that in the absence of any transfers across household-pairs, a household's member income effectively takes on three values $h, \ell$ and $m=\frac{h+\ell}{2}$ with probability $p_{h}=p^{2}$, 
$p_{\ell}=(1-p)^{2}$ and $p_{m}=2 p(1-p)$. So a typical household enjoys utility

$$
\widetilde{v}(2)=p_{h} u(h)+p_{m} u(m)+p_{\ell} u(\ell) .
$$

A sufficient condition for households not to be able to make any transfer to each other is that $(0.19)$

$-(1-\delta) u^{\prime}(h)+\delta\left[-p_{m} p_{h} u^{\prime}(h)+p_{m} p_{h} u^{\prime}(m)-p_{h} p_{\ell} u^{\prime}(h)+p_{h} p_{\ell}-p_{m} p_{\ell} u^{\prime}(h)+p_{h} p_{\ell} \frac{u^{\prime}(h)}{u^{\prime}(m)}\right]<0$.

Let $\ell=1, h=1.488, p=0.5, \delta=0.9, n=4$ and assume a log utility. It can be checked that for these parameters inequality (0.19) is violated. Hence, households would enjoy an insurance gain of $66 \%\left(\frac{\widetilde{v}(2)-v(1)}{\tilde{v}(4)-v(1)} \times 100\right)$. Moreover, in the absence of better enforcement power within households, our simulations reveal that they would enjoy an insurance gain $\left(\frac{v^{*}(4)-v(1)}{\widetilde{v}(4)-v(1)} \times 100\right)$ of $72 \%$. Hence, this implies an additional gain of $16 \%$. 